\title{
Cyclooxygenase-2 expression in non-metastatic triple-negative breast cancer patients
}

\author{
KAILASH MOSALPURIA ${ }^{1}$, CAROLYN HALL ${ }^{1}$, SAVITRI KRISHNAMURTHY ${ }^{2}$, ASHUTOSH LODHI ${ }^{1}$, \\ D. MICHAEL HALLMAN ${ }^{3}$, MARY S. BARANIUK ${ }^{4}$, ANIRBAN BHATTACHARYYA $^{1}$ and ANTHONY LUCCI ${ }^{1}$ \\ Departments of ${ }^{1}$ Surgical Oncology and ${ }^{2}$ Pathology, MD Anderson Cancer Center; Departments of ${ }^{3}$ Epidemiology and \\ ${ }^{4}$ Biostatistics, Health Science Center, School of Public Health, The University of Texas, Houston, TX 77030, USA
}

Received May 8, 2014; Accepted May 29, 2014

DOI: $10.3892 / \mathrm{mco} .2014 .327$

\begin{abstract}
Triple-negative breast cancer (TNBC) is characterised by lack of estrogen receptor (ER), progesterone receptor (PR) and human epidermal growth factor receptor (HER)2/neu gene amplification. TNBC patients typically present at a younger age, with a larger average tumor size, higher grade and higher rates of lymph node positivity compared to patients with ER/PR-positive tumors. Cyclooxygenase (COX)-2 regulates the production of prostaglandins and is overexpressed in a variety of solid tumors. In breast cancer, the overexpression of COX-2 is associated with indicators of poor prognosis, such as lymph node metastasis, poor differentiation and large tumor size. Since both TNBC status and COX-2 overexpression are known poor prognostic markers in primary breast cancer, we hypothesized that the COX-2 protein is overexpressed in the primary tumors of TNBC patients. The purpose of this study was to determine whether there exists an association between TNBC status and COX-2 protein overexpression in primary breast cancer. We prospectively evaluated COX-2 expression levels in primary tumor samples obtained from 125 patients with stage I-III breast cancer treated between February, 2005 and October, 2007. Information on clinicopathological factors was obtained from a prospective database. Baseline tumor characteristics and patient demographics were compared between TNBC and non-TNBC patients using the Chi-square and Fisher's exact tests. In total, $60.8 \%$ of the patients were classified as having ER-positive tumors, $51.2 \%$ were PR-positive, $14.4 \%$ had HER-2/neu amplification and $28.0 \%$ were classified as TNBC. COX-2 overexpression was found in $33.0 \%$ of the patients. TNBC was associated with COX-2 overexpression $(\mathrm{P}=0.009)$, PR expression $(\mathrm{P}=0.048)$ and high tumor grade $(\mathrm{P}=0.001)$. After adjusting for age, menopausal status, body mass index (BMI), lymph node status and neoadjuvant chemotherapy (NACT), TNBC was an independent predictor of $\mathrm{COX}-2$ overexpression $(\mathrm{P}=0.01)$. In
\end{abstract}

Correspondence to: Professor Anthony Lucci, Department of Surgical Oncology, MD Anderson Cancer Center, The University of Texas, 1515 Holcombe Blvd, Houston, TX 77030, USA

E-mail: alucci@mdanderson.org

Key words: cyclooxygenase-2, triple-negative breast cancer conclusion, the association between TNBC and COX-2 overexpression in operable breast cancer supports further investigation into COX-2-targeted therapy for patients with TNBC.

\section{Introduction}

Breast cancer is a heterogeneous disease that is defined and classified using clinical and pathological characteristics, including patient age, tumor size, axillary node involvement, histological grade, estrogen receptor (ER) and progesterone receptor (PR) status and human epidermal growth factor receptor (HER)2/neu (also referred to as ERBB2) amplification (1). Advances in molecular biology techniques have expanded the classical description of breast cancer tumors into distinct subtypes. Three of these subtypes represent ER-negative tumors [triple-negative breast cancer (TNBC), basal-like (BL) and HER2/neu-positive] and two are characterized by ER-positivity (luminal A and B tumors). Tumors classified as luminal A express ER, with or without PR, and lack human HER2 expression; luminal B tumors express ER and HER2, with or without PR expression. BL and triple-negative tumors are highly concordant; both are characterized by a lack of expression of ER, PR and HER2 and are indistinguishable by standard immunohistochemical staining of formalin-fixed and paraffin-embedded samples; therefore, both are classified as TNBC (2). TNBC cases represent 10-30\% of all breast cancers. TNBC patients typically present at a younger age ( $<50$ years), with larger average tumor size, higher grade and higher rates of axillary lymph node positivity compared to ER-positive patients (3-6). TNBC is more prevalent among premenopausal African-American patients compared to postmenopausal African-American and non-African-American patients $(7,8)$.

Due to the lack of estrogen and HER2 expression and the aggressive nature of TNBC, effective management of TNBC patients remains a challenge in the clinical practice. Triple-negative patients are typically treated sequentially with a combination of chemotherapy, surgery and radiation. Triple-negative tumors respond favorably to neoadjuvant chemotherapy (NACT) (9-12) and TNBC patients achieve high pathological complete response ( $\mathrm{pCR}$ ) rates following systemic therapy compared to non-TNBC patients (10). Although there is no reported difference in the rates of local relapse in TNBC vs. non-TNBC patients $(13,14)$, the mean time to recur- 
rence is shorter for TNBCs. TNBC patients typically recur less than 3 years post-diagnosis $(10,13)$ with propensity for distant recurrence to the spinal cord, meninges, brain, liver and lungs (11). The recurrence rate peaks at $\sim 3$ years and diminishes steadily over the next 5 years, accompanied by a very low risk thereafter. Although pCR predicts excellent survival regardless of receptor status, TNBC patients with residual disease following NACT exhibit significantly shorter disease-free and overall survival compared to patients with non-TNBC tumors and residual disease following NACT $(10,13)$. These data highlight the urgent need for improved prognostic tools and novel targeted therapies for TNBC patients.

Cyclooxygenase-2 (COX-2) is an inducible, proinflammatory enzyme that catalyzes key steps in the conversion of arachidonic acid to prostaglandins and thromboxanes. COX-2 expression is induced in tumor cells and is regulated by transcriptional and translational processes that are mediated by cytokines, growth factors and oncogenes (15). Studies using in vitro breast cancer cell lines and in vivo mouse models have demonstrated that COX-2 overexpression plays key roles in tumorigenesis by stimulating epithelial cell proliferation, inhibiting apoptosis, stimulating angiogenesis, increasing multidrug resistance and enhancing cell motility and invasion (15-23). The evidence supporting the role of COX-2 in breast cancer progression has also been demonstrated in clinical studies; patients with COX-2-expressing primary tumors exhibited shortened disease-free and overall survival (24-30).

The majority of the studies employed immunohistochemistry and monoclonal antibodies to assess COX-2 protein expression in primary breast carcinomas and COX-2 positivity was identified in $33-58 \%$ of the cases (24-29,31-34). COX-2 expression is frequently associated with high histological grade and large tumor size $(24-28,30,32,33)$ and, to a lesser degree, negative ER status $(24,26,27,30,31,33)$. The association between COX-2 expression and HER2/neu expression is not conclusive; in two large studies, COX-2 was significantly associated with increased HER2/neu expression (27,33), while no significant association was observed in a number of other studies $(24,25,28,30-32)$. These disparate results emphasize the need for standardized, reproducible assays for COX-2 assessment, which may enable meaningful associations and interpretations.

Triple-negative status and COX-2 expression have both been significantly associated with an unfavorable outcome for non-metastatic breast cancer patients $(30,35)$. The purpose of our study was to determine whether COX-2 protein expression in primary breast cancer is associated with TNBC. Since COX-2 is overexpressed in breast cancer, it may serve as a possible target for chemoprevention in TNBC, for which, apart from systemic chemotherapy, targeted therapy is currently non-existent.

\section{Materials and methods}

Patients. We reviewed the data collected from 125 female patients with stage I-III breast cancer, who were treated at The University of Texas MD Anderson Cancer Center between February, 2005 and October, 2007. The data are derived from an Institutional Review Board (IRB)-approved protocol (DR070276; The University of Texas MD Anderson Cancer Center). The patients were enrolled as a part of two University of Texas MD Anderson Cancer Center IRB-approved research protocols (nos. LAB04-0657 and 04-0698; Principal Investigator, A. Lucci). The patients enrolled in these IRB-approved protocols provided written informed consent for the collection of tissue, blood and bone marrow at the time of their primary surgery for breast cancer. Enrollment was strictly voluntary and the patients did not receive a stipend for participating in this study. The investigators were blinded to individual patient results through the use of a random number system as the unique patient identifier. Patients with bilateral breast cancer or any other malignancy within 5 years of the diagnosis of the current cancer were considered ineligible and were excluded from these studies.

Information on prognostic markers (e.g., ER, PR, HER2 and Ki-67 proliferation index) and other clinical variables, such as age at diagnosis, race, lymph node metastasis, menopausal status, lymphovascular invasion and nuclear grade was obtained from clinical records.

Staging and classification. The primary TNM stage [primary tumor (T), regional nodes (N) and distant metastases (M)] and tumor grade were designated according to the criteria set by the American Joint Commission on Cancer (36) and Black's nuclear grading system (37), respectively. Clinical stage was defined as the TNM stage determined at the time of the first diagnostic procedure confirming the invasive component of the tumor. Axillary lymph node status was determined using ultrasound and fine-needle aspiration. Pathological stage was determined following primary tumor and lymph node removal. Clinical stage was used for analysis for patients who received NACT. The response to NACT was termed as pCR only when there was no evidence of residual invasive disease in the excised tumor and lymph nodes following completion of chemotherapy $(10,38)$.

Immunostaining procedures. The tumor sections were immunostained for ER and PR using previously published procedures (32). The immunostaining results for HER2 were scored as $1+$ when $<10 \%$ of the tumor cells exhibited complete membranous staining; as $2+$ when weak-to-moderate membranous staining was present in $>10 \%$ of the tumor cells; and as 3+ when strong complete membranous staining was present in $>30 \%$ of the tumor cells. All the $2+$ and $3+$ cases were evaluated by fluorescence in situ hybridization for HER2 gene amplification using the PathVysion HER2 DNA probe kit (Abbott Laboratories, Abbott Park, IL, USA). A HER2/chromosome 17 centromere ratio of $>2.2$ was considered as positive for HER2 gene amplification. TNBC was defined by absence of ER and PR expression and HER2 gene amplification in the primary tumor. The tumors were immunostained as previously described and deemed COX-2 positive when $\geq 5 \%$ of the tumor cells immunostained for COX-2 (32). The tumors were considered Ki-67 positive when $\geq 35 \%$ of the tumor cells exhibited Ki-67 staining.

Statistical analysis. The primary tumor characteristics and patient demographics (including pCR) were tabulated and compared between TNBC and non-TNBC patients using the Chi-square and Fisher's exact tests. Odds ratios (ORs) were calculated to assess the association between COX-2 expression and TNBC status. The ORs were adjusted for important 
clinical and pathological prognostic markers [e.g., age, body mass index (BMI), menopausal status, lymph node status and lymphovascular invasion]. The adjusted ORs for COX-2 expression and their 95\% confidence intervals (CIs) were reported using logistic regression. The statistical analyses were performed by statisticians using STATA 13 software (StataCorp, College Station, TX, USA) and $\mathrm{P}<0.05$ was considered to indicate a statistically significant difference.

\section{Results}

Clinicopathological characteristics. The clinical characteristics of the 125 patients and the pathological characteristics of their tumors are presented in Table I. The mean age was 53.4 years (range, 25-92 years) and the mean BMI was $28.6 \mathrm{~kg} / \mathrm{m}^{2}$. Of the 125 patients, $90(72.0 \%)$ were Caucasian, 13/125 (11.0\%) were African-American and 19/125 (15.0\%) were Hispanic. A total of $84(68.0 \%)$ of the 125 patients were postmenopausal.

In total, 39/125 patients (31.2\%) had $\mathrm{T} 1,53 / 125(42.4 \%)$ had T2, 10/125 (8.0\%) had T3 and 23/125 (18.4\%) had T4 tumors; 68/125 patients $(54.4 \%)$ had axillary lymph node metastasis, 60/125 (48.8\%) had high-grade (grade 3) tumors and lymphovascular invasion was present in 42/125 (34.7\%) of the patients. ER, PR and HER2/neu gene amplification were present in $60.8 \%(76 / 125), 51.2 \%(64 / 125)$ and $14.4 \%(18 / 125)$ of the patients, respectively. Based on the information of the three tumor markers, $28.0 \%(35 / 125)$ patients were classified as TNBC. Ki-67 immunostaining is an index of high cellular proliferation, but it is not routinely performed for all patients. Ki-67 data were available for analysis in 51/125 of our sample cohort and $52.9 \%$ (27/51) exhibited a high proliferation index ( $\geq 35 \%$ of tumor cells were Ki-67-positive). A total of 42 patients $(34.7 \%)$ received NACT; 8/125 (6.4\%) achieved pathological partial response or pCR. COX-2 expression assessment was available for 106 patients in this study. Using a 5\% threshold for COX-2 expression, 35/106 (33.0\%) patients had primary tumors that were $\mathrm{COX}-2$-positive.

Association of COX-2 expression with clinicopathological characteristics. The unadjusted ORs between COX-2 expression and primary tumor characteristics are shown in Table IIA. COX-2-expressing tumors ( $\geq 5 \%$ of the cells expressing COX-2) were three times more likely to be TNBC (OR=3.34, 95\% CI: $1.40-8.22 ; \mathrm{P}=0.009)$ and four times more likely to be high-grade $(\mathrm{OR}=4.09,95 \% \mathrm{CI}$ : $1.58-10.82 ; \mathrm{P}=0.001)$ compared to COX-2-negative tumors (COX-2 expression in $<5 \%$ of the cells). No significant associations were observed between COX-2 expression and ER positivity $(\mathrm{P}=0.10), \mathrm{HER} 2 /$ neu gene amplification $(\mathrm{P}=0.18)$, or Ki-67 index $(\mathrm{P}=0.09)$. However, $\mathrm{PR}$ positivity was associated with $\mathrm{COX}-2$ expression $(\mathrm{OR}=0.43$, 95\% CI: 0.19-0.99; $\mathrm{P}=0.048)$. After adjusting for age, BMI, menopausal status, lymph node status and lymphovascular invasion, the multivariate analysis demonstrated that TNBC patients were more likely to exhibit COX-2 expression (OR=3.48, 95\% CI: 1.28-9.44; $\mathrm{P}=0.01$ ) (Table IIB).

Association of TNBC with clinicopathological characteristics. In our study, TNBC was associated with high tumor grade $(\mathrm{OR}=8.89$, 95\% CI: 3.12-28.55; $\mathrm{P}<0.001)$ (Table IIIA). We
Table I. Clinicopathological characteristics of the 125 breast cancer patients.

\begin{tabular}{|c|c|}
\hline Characteristics & $\begin{array}{l}\text { Number of patients, no. (\%) } \\
\qquad(\mathrm{n}=125)\end{array}$ \\
\hline Mean age, years & 53.4 \\
\hline Mean BMI, $\mathrm{kg} / \mathrm{m}^{2}$ & 28.6 \\
\hline \multicolumn{2}{|l|}{ Race } \\
\hline Caucasian & $90(72.0)$ \\
\hline African-American & $13(11.0)$ \\
\hline Hispanic & $19(15.0)$ \\
\hline Other & $3(2.0)$ \\
\hline Postmenopausal & $84(68.0)$ \\
\hline \multicolumn{2}{|l|}{ Tumor size } \\
\hline $\mathrm{T} 1^{\mathrm{a}}$ & $39(31.2)$ \\
\hline $\mathrm{T} 2^{\mathrm{b}}$ & $53(42.4)$ \\
\hline $\mathrm{T} 3^{\mathrm{c}}$ & $10(8.0)$ \\
\hline $\mathrm{T} 4^{\mathrm{d}}$ & $23(18.4)$ \\
\hline Axillary LN metastasis & $68(54.4)$ \\
\hline High-grade tumor ${ }^{\mathrm{e}}$ & $60(48.8)$ \\
\hline LVI & $42(34.7)$ \\
\hline ER & $76(60.8)$ \\
\hline PR & $64(51.2)$ \\
\hline HER-2/neu amplification & 18 (14.4) \\
\hline $\mathrm{TNBC}^{\mathrm{f}}$ & $35(28.0)$ \\
\hline NACT & $42(34.7)$ \\
\hline COX-2 expression $(n=106)^{g}$ & $35(33.0)$ \\
\hline Ki-67 $(n=51)^{\mathrm{h}}$ & $27(52.9)$ \\
\hline
\end{tabular}

${ }^{\mathrm{a}} \leq 2 \mathrm{~cm} .{ }^{\mathrm{b}}>2$ and $\leq 5 \mathrm{~cm} .{ }^{\mathrm{c}}>5 \mathrm{~cm}$. ${ }^{\mathrm{d}}$ Any tumor size, but extending to the overlying skin or chest wall. ${ }^{\mathrm{e}}$ Grade 3 . ${ }^{\mathrm{f}} \mathrm{Absence}$ of ER and PR positivity and HER 2 gene amplification. ${ }^{9}$ Presence of COX-2 expression in $\geq 5 \%$ of cells as determined by immunohistochemistry. ${ }^{\mathrm{h}} \geq 35 \%$ of tumor cells exhibiting Ki-67 staining. BMI, body mass index; LN, lymph nodes; LVI, lymphovascular invasion; ER, estrogen receptor; $\mathrm{PR}$, progesterone receptor; HER2, human epidermal growth factor receptor 2; TNBC, triple-negative breast cancer; NACT, neoadjuvant chemotherapy; COX-2, cyclooxygenase-2.

identified a negative association between TNBC and lymphovascular invasion $(\mathrm{OR}=0.43,95 \% \mathrm{CI}$ : $0.06-0.73 ; \mathrm{P}=0.006)$. We found no significant association between TNBC and positive axillary lymph node status $(\mathrm{OR}=1.16,95 \% \mathrm{CI}$ : 0.49-2.78; $\mathrm{P}=0.70)$, or $\mathrm{pCR}(\mathrm{OR}=2.77,95 \% \mathrm{CI}: 0.48-15.72 ; \mathrm{P}=0.15)$ (Table IIIA). The multivariate analysis demonstrated that TNBC was significantly associated with high tumor grade $(\mathrm{OR}=6.30,95 \%$ CI: 2.15-18.41; $\mathrm{P}<0.001)$ (Table IIIB).

\section{Discussion}

Previous studies demonstrated that COX-2 expression $(24,25,27-29,35,39,40)$ and TNBC $(7,8,10,13,14,41-44)$ are independent predictors of poor prognosis in stage I-III breast cancer. Based on this information, we hypothesized 
Table II. Association of COX-2 expression with clinicopathological characteristics.

A, Unadjusted measures of association of COX-2 expression with ER, PR, HER2 gene amplification, TNBC, high tumor grade and Ki-67.

\begin{tabular}{lccl}
\hline Characteristics & OR & $95 \%$ CI & P-value \\
\hline ER & 0.49 & $0.22-1.15$ & 0.10 \\
PR & 0.43 & $0.19-0.99$ & $0.048^{\mathrm{a}}$ \\
HER2 & 0.23 & $0.03-1.93$ & $0.18^{\mathrm{b}}$ \\
TNBC & 3.34 & $1.40-8.22$ & $0.009^{\mathrm{a}}$ \\
High grade & 4.09 & $1.58-10.82$ & $0.001^{\mathrm{a}}$ \\
Ki-67 & 2.78 & $0.71-11.29$ & 0.09 \\
\hline
\end{tabular}

B, Adjusted ORs from a multivariate logistic regression analysis with COX-2 expression as the dependent variable.

\begin{tabular}{lccc}
\hline Characteristics & OR & $95 \%$ CI & P-value \\
\hline Age & 0.99 & $0.94-1.04$ & 0.66 \\
BMI & 0.95 & $0.88-1.02$ & 0.16 \\
Postmenopausal $^{\text {LN status }}{ }^{\mathrm{c}}$ & 2.03 & $0.56-7.35$ & 0.28 \\
LVI & 2.22 & $0.89-5.53$ & 0.09 \\
TNBC & 1.25 & $0.43-3.60$ & 0.68 \\
& 3.48 & $1.28-9.44$ & $0.01^{\mathrm{a}}$ \\
\hline
\end{tabular}

${ }^{a}$ Denotes statistical significance $(\mathrm{P}<0.05)$. ${ }^{\mathrm{b}}$ Fisher's exact test. ${ }^{\mathrm{c}}$ Presence of axillary lymph node metastasis. COX-2, cyclooxygenase-2; ER, estrogen receptor; PR, progesterone receptor; HER2, human epidermal growth factor receptor 2; TNBC, triple-negative breast cancer; OR, odds ratio; CI, confidence interval; BMI, body mass index; LN, lymph node; LVI, lymphovascular invasion.

that increased COX-2 expression may be associated with TNBC status. A total of $33.0 \%$ of our sample cohort exhibited COX-2 expression (using the 5\% threshold), which is similar to the results of previous studies (24-29,31-34). In congruence with previously published reports, we observed a significant association between COX-2 expression and high tumor grade $(26-28,30,32,33)$ and also identified a significant association between COX-2 expression and TNBC. The association between TNBC status and high tumor grade $(\mathrm{OR}=8.89$, 95\% CI: 3.12-28.55; $\mathrm{P}<0.001)$ was in agreement with results of larger studies $(12,13,15,16,18,22)$. We did not identify a significant association between TNBC and positive axillary lymph node status, as reported in several $(8,11,44)$, but not all $(8,10,13)$ studies.

Preliminary reports indicated that COX-2 expression predicts outcome in early-stage (stage I/II) and node-negative patients $(25,28)$. Although we did not determine the prognostic significance of COX-2 expression in the present study, it is intriguing to consider the potential COX-2-mediated mechanisms involved in disease progression. In addition to the well-documented in vitro and in vivo COX-2-mediated tumorigenic/angiogenic effects, COX-2 expression has also been shown to correlate with increased resistance to radia-
Table III. Association of TNBC with clinicopathological characteristics.

A, Unadjusted measures of association of TNBC with high tumor grade, LVI, LN status and pCR.

\begin{tabular}{lccc}
\hline Characteristics & OR & \multicolumn{1}{c}{$95 \%$ CI } & P-value \\
\hline High grade & 8.89 & $3.12-28.55$ & $<0.001^{\text {a }}$ \\
LVI & 0.43 & $0.06-0.73$ & $0.006^{\mathrm{a}}$ \\
LN status & 1.16 & $0.49-2.78$ & 0.70 \\
pCR & 2.77 & $0.48-15.72$ & 0.15 \\
\hline
\end{tabular}

B, Adjusted ORs from a multivariate logistic regression analysis with TNBC as the dependent variable.

\begin{tabular}{lccr}
\hline Characteristics & OR & $95 \% \mathrm{CI}$ & P-value \\
\hline High grade & 6.30 & $2.15-18.41$ & $<0.001^{\mathrm{a}}$ \\
LVI & 0.28 & $0.08-0.92$ & $0.036^{\mathrm{a}}$ \\
LN status & 1.53 & $0.49-4.76$ & 0.46 \\
pCR & 2.38 & $0.24-24.14$ & 0.46
\end{tabular}

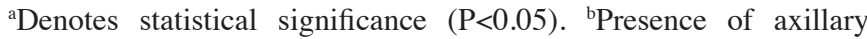
lymph node metastasis. TNBC, triple-negative breast cancer; LVI, lymphovascular invasion; LN, lymph node; pCR, pathological complete response; OR, odds ratio; CI, confidence interval.

tion therapy $(45,46)$ and poorer response to chemotherapeutic agents (47). In addition, a 2009 report published by our group demonstrated that COX-2 expression in the primary tumor predicted the presence of bone marrow micrometastasis; the bone marrow tumor cells also exhibited COX-2 overexpression (32), suggesting that, in addition to tumor cell dissemination to distant sites by lymphatic spread, COX-2-expressing tumor cells may disseminate through the hematogenous route.

It is difficult to draw statistical conclusions regarding the association of COX-2 positivity with different races or ethnicities and adjust for potential confounders. Our patient sample size was limited to patients with non-metastatic breast cancer who were consented and treated at a single tertiary care hospital. Our findings should now be evaluated in a multicenter setting with larger patient samples. The cross-sectional nature of this study may lead to some bias due to the unequal distribution of confounders. This study was a secondary analysis of data; therefore, our results must be interpreted with caution, since the primary study was designed to assess the association between COX-2 expression in the primary tumor and detection of microscopic disease in the bone marrow and peripheral blood at the time of primary surgery. It is possible that NACT resulted in widespread changes within the tumor bed that may have interfered with the detection of COX-2 expression in the tissue acquired at the time of surgery. This scenario may be avoided by measuring COX-2 expression in tissues obtained prior to chemotherapy initiation.

In conclusion, we report that COX-2, an extensively investigated marker of poor prognosis in patients with non-metastatic breast cancer, is associated with TNBC and high tumor grade. 
Due to the aggressive nature of TNBC, assessing the COX-2 expression in TNBC patients may provide valuable prognostic information and assist in identifying those TNBC patients at higher risk for recurrence. Since TNBC patients have only limited treatment options, clinical trials investigating COX-2 suppression using COX-2 inhibitors are required.

\section{Acknowledgements}

This study was supported by the National Institutes of Health, grant no. R21-DK067682 (A.L.); the Department of Defense Breast Cancer Research Program (DAMD 17-03-01-0669); the Society of Surgical Oncology Clinical Investigator Award; the MD Anderson Institute for Personalized Cancer Therapy (A.L.); and a grant from the State of Texas Rare and Aggressive Breast Cancer Research program.

\section{References}

1. Perou CM, Sorlie T, Eisen MB, et al: Molecular portraits of human breast tumours. Nature 406: 747-752, 2000.

2. Bertucci F,Finetti P,Cervera N, et al: How basal are triple-negative breast cancers? Int J Cancer 123: 236-240, 2008.

3. Sorlie T, Perou CM, Tibshirani R, et al: Gene expression patterns of breast carcinomas distinguish tumor subclasses with clinical implications. Proc Natl Acad Sci USA 98: 10869-10874, 2001.

4. Sorlie T, Tibshirani R, Parker J, et al: Repeated observation of breast tumor subtypes in independent gene expression data sets. Proc Natl Acad Sci USA 100: 8418-8423, 2003.

5. Sotiriou C, Neo SY, McShane LM, et al: Breast cancer classification and prognosis based on gene expression profiles from a population-based study. Proc Natl Acad Sci USA 100: 10393-10398, 2003.

6. van 't Veer LJ, Dai H, van de Vijver MJ, et al: Gene expression profiling predicts clinical outcome of breast cancer. Nature 415: 530-536, 2002.

7. Carey LA, Perou CM, Livasy CA, et al: Race, breast cancer subtypes and survival in the Carolina Breast Cancer Study. JAMA 295: 2492-2502, 2006.

8. Lund MJ, Trivers KF, Porter PL, et al: Race and triple negative threats to breast cancer survival: a population-based study in Atlanta, GA. Breast Cancer Res Treat 113: 357-370, 2009.

9. Carey LA, Dees EC, Sawyer L, et al: The triple negative paradox: primary tumor chemosensitivity of breast cancer subtypes. Clin Cancer Res 13: 2329-2334, 2007.

10. Liedtke C, Mazouni C, Hess KR, et al: Response to neoadjuvant therapy and long-term survival in patients with triple-negative breast cancer. J Clin Oncol 26: 1275-1281, 2008.

11. Rakha EA, El-Sayed ME, Green AR, Lee AH, Robertson JF and Ellis IO: Prognostic markers in triple-negative breast cancer. Cancer 109: 25-32, 2007.

12. Rouzier R, Perou CM, Symmans WF, et al: Breast cancer molecular subtypes respond differently to preoperative chemotherapy. Clin Cancer Res 11: 5678-5685, 2005.

13. Dent R, Trudeau M, Pritchard KI, et al: Triple-negative breast cancer: clinical features and patterns of recurrence. Clin Cancer Res 13: 4429-4434, 2007.

14. Haffty BG, Yang Q, Reiss M, et al: Locoregional relapse and distant metastasis in conservatively managed triple negative early-stage breast cancer. J Clin Oncol 24: 5652-5657, 2006.

15. Singh-Ranger G, Salhab M and Mokbel K: The role of cyclooxygenase-2 in breast cancer: review. Breast Cancer Res Treat 109: 189-198, 2008

16. Basu GD, Liang WS, Stephan DA, et al: A novel role for cyclooxygenase- 2 in regulating vascular channel formation by human breast cancer cells. Breast Cancer Res 8: R69, 2006.

17. Basu GD, Pathangey LB, Tinder TL, Lagioia M, Gendler SJ and Mukherjee P: Cyclooxygenase-2 inhibitor induces apoptosis in breast cancer cells in an in vivo model of spontaneous metastatic breast cancer. Mol Cancer Res 2: 632-642, 2004.

18. Hoellen F, Kelling K, Dittmer C, Diedrich K, Friedrich M and Thill M: Impact of cyclooxygenase-2 in breast cancer. Anticancer Res 31: 4359-4367, 2011.

19. Jendrossek V: Targeting apoptosis pathways by celecoxib in cancer. Cancer Lett 332: 313-324, 2013.
20. Liu B, Qu L and Tao H: Cyclo-oxygenase 2 up-regulates the effect of multidrug resistance. Cell Biol Int 34: 21-25, 2009.

21. Sahin M, Sahin E and Gumuslu S: Cyclooxygenase-2 in cancer and angiogenesis. Angiology 60: 242-253, 2009.

22. Singh B, Berry JA, Shoher A, Ramakrishnan V and Lucci A: COX-2 overexpression increases motility and invasion of breast cancer cells. Int J Oncol 26: 1393-1399, 2005.

23. Thill M, Fischer D, Becker S, et al: Prostaglandin metabolizing enzymes in correlation with vitamin $\mathrm{D}$ receptor in benign and malignant breast cell lines. Anticancer Res 29: 3619-3625, 2009.

24. Denkert C, Winzer KJ, Muller BM, et al: Elevated expression of cyclooxygenase-2 is a negative prognostic factor for disease free survival and overall survival in patients with breast carcinoma. Cancer 97: 2978-2987, 2003.

25. Haffty BG, Yang Q, Moran MS, Tan AR and Reiss M: Estrogen-dependent prognostic significance of cyclooxygenase-2 expression in early-stage invasive breast cancers treated with breast-conserving surgery and radiation. Int J Radiat Oncol Biol Phys 71: 1006-1013, 2008 .

26. Kim HS, Moon HG, Han W, et al: COX2 overexpression is a prognostic marker for stage III breast cancer. Breast Cancer Res Treat 132: 51-59, 2012.

27. Ristimaki A, Sivula A, Lundin J, et al: Prognostic significance of elevated cyclooxygenase- 2 expression in breast cancer. Cancer Res 62: 632-635, 2002.

28. Schmitz KJ, Callies R, Wohlschlaeger J, et al: Overexpression of cyclo-oxygenase-2 is an independent predictor of unfavourable outcome in node-negative breast cancer, but is not associated with protein kinase $\mathrm{B}(\mathrm{Akt})$ and mitogen-activated protein kinase (ERK1/2, p38) activation or with Her-2/neu signalling pathways. J Clin Pathol 59: 685-691, 2006.

29. Spizzo G, Gastl G, Wolf D, et al: Correlation of COX-2 and Ep-CAM overexpression in human invasive breast cancer and its impact on survival. Br J Cancer 88: 574-578, 2003.

30. van Nes JG, de Kruijf EM, Faratian D, et al: COX2 expression in prognosis and in prediction to endocrine therapy in early breast cancer patients. Breast Cancer Res Treat 125: 671-685, 2011.

31. Kargi A, Uysal M, Bozcuk H, Coskun HS, Savas B and Ozdogan M: The importance of COX-2 expression as prognostic factor in early breast cancer. J BUON 18: 579-584, 2013.

32. Lucci A, Krishnamurthy S, Singh B, et al: Cyclooxygenase-2 expression in primary breast cancers predicts dissemination of cancer cells to the bone marrow. Breast Cancer Res Treat 117: 61-68, 2009.

33. Wulfing P, Diallo R, Muller C, et al: Analysis of cyclooxygenase-2 expression in human breast cancer: high throughput tissue microarray analysis. J Cancer Res Clin Oncol 129: 375-382, 2003

34. Zhou L, Li K, Luo Y, et al: Novel prognostic markers for patients with triple-negative breast cancer. Hum Pathol 44: 2180-2187, 2013.

35. Witton CJ, Hawe SJ, Cooke TG and Bartlett JM: Cyclooxygenase 2 (COX2) expression is associated with poor outcome in ER-negative, but not ER-positive, breast cancer. Histopathology 45: 47-54, 2004.

36. Edge S, Byrd DR, Compton CC, Fritz AG, Greene FL and Trotti A (eds): AJCC Cancer Staging Manual. 7th edition. Springer, New York, NY, pp 421, 2010.

37. Black MM and Speer FD: Nuclear structure in cancer tissues. Surg Gynecol Obstet 105: 97-102, 1957.

38. Mazouni C, Peintinger F, Wan-Kau S, et al: Residual ductal carcinoma in situ in patients with complete eradication of invasive breast cancer after neoadjuvant chemotherapy does not adversely affect patient outcome. J Clin Oncol 25: 2650-2655, 2007.

39. O'Connor JK, Avent J, Lee RJ, Fischbach J and Gaffney DK: Cyclooxygenase-2 expression correlates with diminished survival in invasive breast cancer treated with mastectomy and radiotherapy. Int J Radiat Oncol Biol Phys 58: 1034-1040, 2004.

40. Zerkowski MP, Camp RL, Burtness BA, Rimm DL and Chung GG: Quantitative analysis of breast cancer tissue microarrays shows high cox- 2 expression is associated with poor outcome. Cancer Invest 25: 19-26, 2007.

41. Bauer KR, Brown M, Cress RD, Parise CA and Caggiano V: Descriptive analysis of estrogen receptor (ER)-negative, progesterone receptor (PR)-negative and HER2-negative invasive breast cancer, the so-called triple-negative phenotype: a population-based study from the California cancer Registry. Cancer 109: 1721-1728, 2007. 
42. Bhargava R, Beriwal S, Dabbs DJ, et al: Immunohistochemical surrogate markers of breast cancer molecular classes predicts response to neoadjuvant chemotherapy: a single institutional experience with 359 cases. Cancer 116: 1431-1439, 2010

43. Morris GJ, Naidu S, Topham AK, et al: Differences in breast carcinoma characteristics in newly diagnosed African-American and Caucasian patients: a single-institution compilation compared with the National Cancer Institute's Surveillance, Epidemiology and End Results database. Cancer 110: 876-884, 2007.

44. Tischkowitz M, Brunet JS, Begin LR, et al: Use of immunohistochemical markers can refine prognosis in triple negative breast cancer. BMC Cancer 7: 134, 2007.
45. Davis TW, Hunter N, Trifan OC, Milas L and Masferrer JL: COX-2 inhibitors as radiosensitizing agents for cancer therapy. Am J Clin Oncol 26: S58-S61, 2003.

46. Raju U, Ariga H, Dittmann K, Nakata E, Ang KK and Milas L: Inhibition of DNA repair as a mechanism of enhanced radioresponse of head and neck carcinoma cells by a selective cyclooxygenase-2 inhibitor, celecoxib. Int J Radiat Oncol Biol Phys 63: 520-528, 2005.

47. Lau L, Hansford LM, Cheng LS, et al: Cyclooxygenase inhibitors modulate the p53/HDM2 pathway and enhance chemotherapy-induced apoptosis in neuroblastoma. Oncogene 26: 1920-1931, 2007. 